Acta Genet Med Gemellol 45: 199-204 (1996)

(C) 1996 by The Mendel Institute

\title{
The Mouse Xist Gene: a Model for Studying the Gametic Imprinting Phenomenon
}

\author{
M. Zuccotti ${ }^{1,2}$, M. Monk ${ }^{1}$ \\ ${ }^{1}$ Molecular Embriology Unit, Institute of Child Health, London, United Kingdom; ' Dipar- \\ timento Biologia Animale e Centro Studi per l'Istochimica del CNR, Pavia, Italia
}

\section{INTRODUCTION}

In mammals, normal embryonic development requires differential genomic imprinting of male and female gametes [1,2]. Many investigations have been directed towards the understanding of the molecular mechanisms of imprinting and the timing of establishment of the imprint during gametogenesis and its erasure during development.

Methylation is the focus of many of these studies as it has been known for some time that this epigenetic modification of the DNA correlates with the status of gene activity. So far, five imprinted genes, expressed from only one of the parental alleles, have been found to be differentially methylated in somatic tissue: mouse Igf2 [3] and Xist [4] and human $S N R P N[5,6]$ expressed from the paternal allele; mouse $\operatorname{Igf} 2 r$ [7] and $H 19$ [8, 9] expressed from the maternal allele. However, so far, a gametic methylation imprint has been detected for only two of these genes: in an intron region of mouse $\operatorname{Ig} f 2 r$ [7], and in the promoter region [10] and the first exon [11] of the Xist (X-inactivation-specific transcript $[12,13]$ gene.

The data accumulated for the Xist gene, during different phases of gametogenesis and development, provides the most comprehensive story about the role of methylation as a primary gametic imprint, and on the timing of its establishment during gametogenesis and erasure during development. Methylation studies have now been performed during oogenesis and spermatogenesis [Norris et al., 1994; 11] and in mature gametes and during early stages of development $[10,11]$. In addition, expression of the gene has been described during gametogenesis [14-16] and throughout early development [4-17].

The Xist gene, located in the region of the $\mathrm{X}$ inactivation centre (Xic) on the $\mathrm{X}$ chromosome $[18,19]$ is believed to be involved in the inactivation of the $\mathrm{X}$ chromosome in 
female mouse embryo development. Inactivation first occurs in the extraembryonic tissues of the blastocyst and later in the embryonic cells after implantation [20]. Inactivation is random in the embryonic cells (either the paternal or maternal $\mathrm{X}$ chromosome is inactivated), whereas the paternal $X$ chromosome is preferentially inactivated in the extraembryonic trophectoderm and primary endoderm [21-23]. Preferential inactivation of the paternal $\mathrm{X}$ chromosome implies the existence of a mark (imprint) distinguishing the paternal and maternal X chromosomes. The identification of the Xic [18], and the discovery of the Xist gene $[12,13]$ transcribed only from the inactive $X$ chromosome, shifted the quest for the imprint to a more molecular level.

The question that we addressed when we began our study was the following: is differential methylation the primary gametic imprint distinguishing the paternal and maternal Xist alleles in such a way that preferential paternal $\mathrm{X}$ inactivation occurs at the blastocyst stage?

Our investigations [10] and those of Ariel et al. [11] have shown that two CpG sites in the promoter region and six sites in the first exon are unmethylated in sperm but methylated in eggs. These differences in methylation correlate with the exclusive expression of the paternal allele in early development. Moreover, the differential methylation of the parental Xist alleles appears to be maintained throughout preimplantation development until the time of inactivation of the paternal $X$ chromosome in the extraembryonic lineages. In this review, we discuss these results, together with previous studies, to provide an overall picture of the imprinting of the Xist gene in development.

\section{Xist expression and methylation: a cronology of events}

Figure 1 is a diagrammatic representation of the data presented below on Xist expression and Xist methylation in gametogenesis and throughout preimplantation development.

\section{Spermatogenesis}

Studies on expression have shown that Xist transcripts are not detected in prospermatogonia cells at $15.5,18.5$ and 21.5 d.p.c. Expression is first detected at the spermatogonia stage in 6-day old male mice, continues in primary spermatocytes, and then at a diminished level in round spermatids, to finally cease in mature spermatozoa [14-16].

Studies on the methylation pattern of Xist during spermatogenesis reveal a striking correlation in methylation of certain CpG sites within the first exon and Xist expression. $X i s t$ is methylated (and not expressed) in prospermatogonia cells and demethylation occurs in spermatogonial cells with the initiation of expression [11]. The demethylated status is maintained throughout all further stages of spermatogenesis, in spermatids and in mature sperm. The lack of Xist expression in postmeiotic male germ cells is associated with the overall cessation of gene transcription occurring at this stage.

\section{Oogenesis}

The Xist transcript has been detected in female primordial germ cells (PGCs) at 12.5 d.p.c. Then, as the oocyte enters meiosis, $X$ chromosome reactivation occurs and Xist 


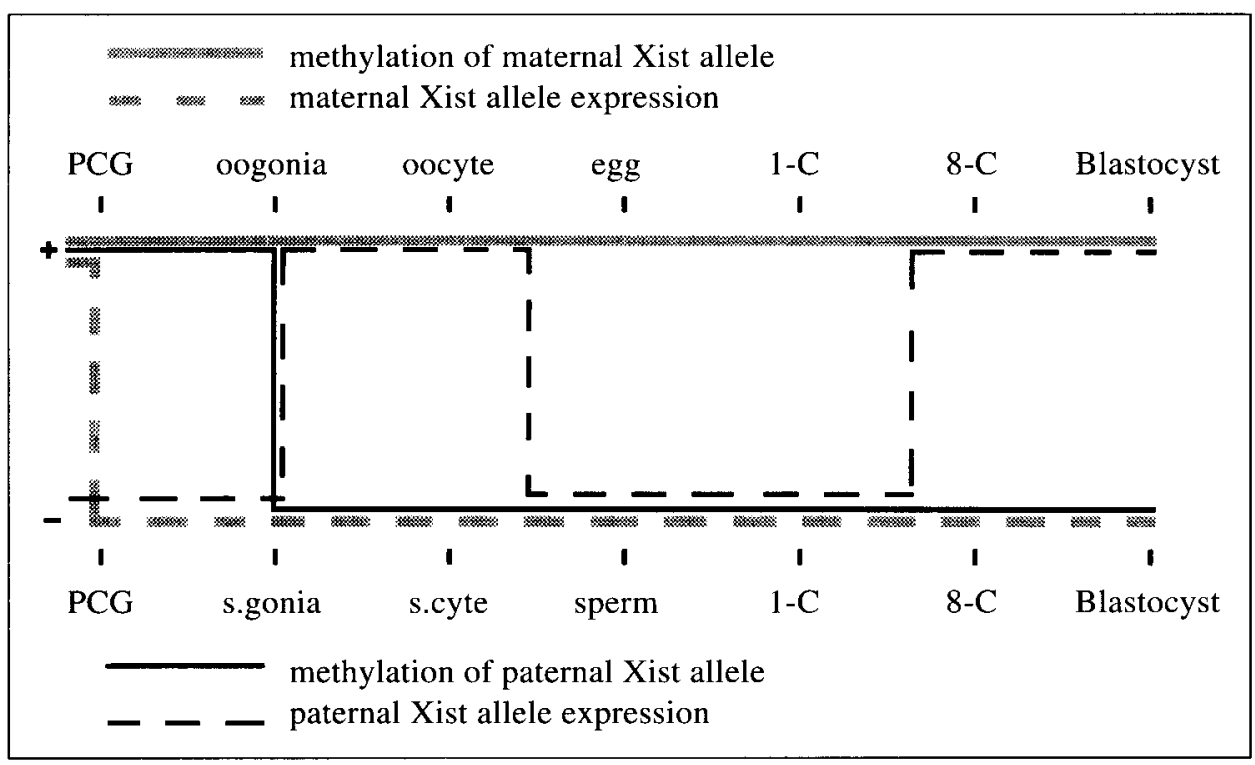

Fig. 1 - Diagrammatic representation of the patterns of expression and methylation of the Xist gene throughout spermatogenesis, oogenesis and preimplantation embryonic development. PGC $=$ Primordial germ cell.

expression terminates (13.5 d.p.c.) [15]. At the time of cessation of expression, female PGCs are methylated at the CpG sites studied by Ariel et al. [11] in the first exon. Methylation of these $\mathrm{CpG}$ sites is maintained in germinal vesicle oocytes and in mature oocytes.

\section{Gametes}

Zuccotti and Monk [10] and Ariel et al. [11] examined the pattern of methylation of a number of $\mathrm{CpG}$ sites in the Xist gene in sperm and eggs. Both groups found a difference in methylation status which correlates with the differential expression of the paternal allele during preimplantation development [4]. Zuccotti and Monk [10] looked at the promoter region close to the major transcription site and identified two CpG sites that are methylated in the female gamete, but unmethylated in the male gamete. The other three $\mathrm{CpG}$ sites studied within the promoter region are methylated in both sperm and eggs. The two differentially methylated sites are located in a promoter domain where the interaction between DNA and putative transcription factors is likely to occur which suggests that differential methylation is the regulatory imprint that governs protein binding. An additional six CpG sites in the first exon of the Xist gene were analysed by Ariel et al. [11]. These sites are also unmethylated in sperm and methylated in eggs. The absence of methylation in sperm is likely to be causal to the exclusive expression of the paternal allele in the 4-cell embryo. 


\section{Preimplantation embryos}

Expression of the paternal Xist allele in the 4-cell-stage embryo occurs exclusively from the paternal allele, and continues to the blastocyst stage [4]. Expression of the female allele is first seen in the embryo at 6.5 d.p.c. [17]. The exclusive expression of the paternal allele may be explained by the observation that the methylation of the maternal allele, both in the promoter region [10] and in the first exon [11], is maintained throughout preimplantation development, while the paternal alleles remain unmethylated.

\section{Erasure of imprinting}

Figure 2 shows maintenance of the imprint to the blastocyst and erasure of the imprint after implantation. The paternal allele enters the zygote unmethylated at specific CpG sites, whereas the maternal alleles are methylated at these sites. The differential pattern of methylation of the two alleles is maintained to the blastocyst stage. Thus, the imprint survives the overall demethylation process which begins from the 8-cell stage [Monk et al., 1987] and marks the paternal $X$ chromosome for inactivation in the extraembryonic lineages of the blastocyst. At the time of $\mathrm{X}$ inactivation in the embryonic precursor cells at implantation, we assume that the differential methylation has been erased so that now $\mathrm{X}$ inactivation is random in these cells.

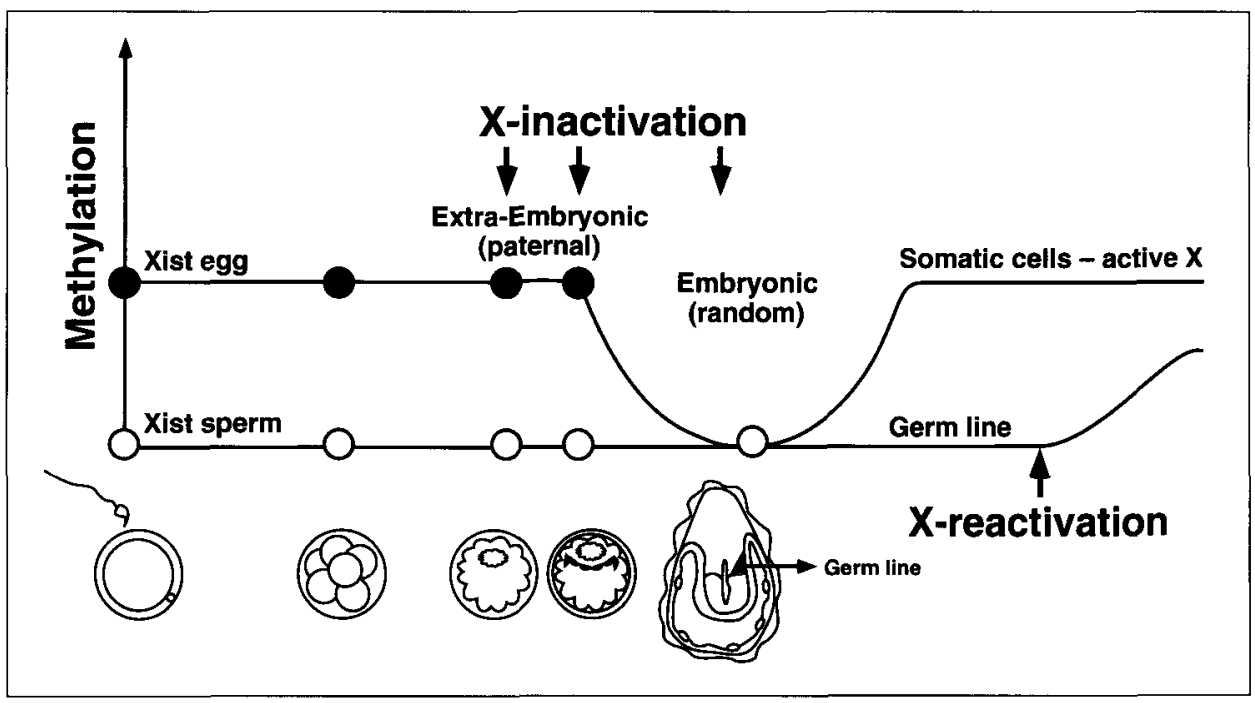

Fig. 2 - Diagrammatic representation of the maintenance of the differentially methylated sites during preimplantation development of the female embryo. In this model, the erasure of the methylation imprint occurs before random inactivation in fetal precursors cells and the delineation of the germ line. De novo methylation then distinguishes the active $\mathrm{X}$ chromosome in somatic cells soon after embryo implantation. 


\section{CONCLUDING REMARKS}

This paper reviews data showing a striking correlation between Xist gene expression and its pattern of methylation during the different stages of both male and female gametogenesis and throughout preimplantation development. Differential methylation of $\mathrm{CpG}$ sites in the Xist promoter and first exon in sperm and eggs regulates differential expression of parental alleles in development.

Further investigations will determine the effect of methylation on the binding of known protein transcription factors to Xist DNA and will attempt the isolation of as yet unknown proteins showing differential binding to the unmethylated and methylated Xist promoter and exon regions. We expect that the Xist gene will continue to be a good model for understanding the genomic imprinting phenomenon. However, it must be borne in mind that other imprinted genes may have different molecular mechanisms of imprinting and a different chronology of establishment and erasure of the imprinted signal.

\section{REFERENCES}

1. Surani MAH et al.: Development of reconstituted mouse eggs suggests imprinting of the genome during gametogenesis. Nature 1984; 308: 548-550.

2. McGrath J, Solter D: Completion of mouse embryogenesis requires both maternal and paternal genomes. Cell 1984; 37: 179-183.

3. Brandeis $M$, et al.: The ontogeny of allele-specific methylation associated with imprinted genes in the mouse. EMBO J 1993; 12: 3669-3677.

4. Kay GF. et al.: Imprinting and X chromosome counting mechanisms determine Xist expression in early mouse development. Cell 1994; 77: 639-650.

5. Glenn CC et al.: Functional imprinting and epigenetic modification of the human $S N R P N$ gene. Hum Mol Genet 1993; 2: 2001-2005.

6. Sutcliffe JS et al.: Deletions of differentially methylated $\mathrm{CpG}$ island at the $S N R P N$ gene define a putative imprinting control region. Nat Genet 1994; 8: 52-58.

7. Stoger $\mathrm{R}$ et al.: Maternal specific methylation of the imprinted mouse Igf $2 r$ locus identifies the expressed locus as carrying the imprinted signal. Cell 1993; 73:61-71.

8. Bartolomei MS et al.: Epigenetic mechanisms underlying the imprinting of the mouse $\mathrm{H} 19$ gene. Genes Dev 1993; 7: 1663-1673.

9. Ferguson-Smith AC et al.: Parental-origin specific epigenetic modification of the mouse $H 19$ gene. Nature 1993; 362:751-755.

10. Zuccotti M, Monk M: Methylation of the mouse Xist gene in sperm and eggs correlates with imprinted Xist expression and paternal X inactivation. Nat Genet 1995; 9: 316-320.

11. Ariel $\mathrm{M}$ et al.: Gamete specific methylation correlates with imprinting of the murine Xist gene. Nat Genet 1995; à: 312-315.3.

12. Borsani $G$ et al.: Characterisation of a murine gene expressed from the inactive $X$ chromosome. Nature 1991; 351: 325-329.

13. Brockdorff $\mathrm{N}$. et al.: Conservation of position and exclusive expression of mouse Xist from the inactive X chromosome. Nature 1991; 351: 329-331.

14. Salido EC et al.: Expression of the $\mathrm{X}$ inactivation-associated gene Xist during spermatogenesis. Nat Genet 1992; 2: 196-199.

15. McCarrey JR, Dilworth DD: Expression of Xist in mouse germ cells correlates with X-chromosome inactivation. Nat Genet 1992; 2: 200-203. 


\section{M. Zuccotti et al.}

16. Richler $\mathrm{C}$ et al.: $\mathrm{X}$ inactivation in mammalian testis is correlated with inactive $\mathrm{X}$-specific transcription. Nat Genet 1992; 2: 192-195.

17. Kay GF et al:: Expression of Xist during mouse development suggests a role in the initiation of $X$ chromosome inactivation. Cell 1993; 72: 171-182.

18. Brown DM: Xist and the mapping of the X chromosome inactivation centre. Bio essays 1991; 13: 607-612.

19. Heard E, Avner P: Role play in X inactivation. Hum Mol Genet 1994; 3: 148 1-1485.

20. Monk M, Harper M: Sequential X chromosome inactivation coupled with cellular differentiation in early mouse embryos. Nature $1979 ; 281: 311-313$.

21. Takagi N, Sasaki M: Preferential expression of the paternally derived X chromosome in the extraeembryonic membranes of the mouse. Nature 1975; 256: 640-642.

22. West JD et al.: Preferential expression of the maternally derived $X$ chromosome in the mouse yolk sac. Cell 1977; 12: 873-882.

23. Harper Ml et al.: Preferential paternal X-inactivation in extraembryonic tissues of early mouse embryos. J Embryol Exp Morphol 1982; 67: 127-135.

Correspondence: Maurizio Zuccotti, Molecular Embriology Unit, Institute of Child Health, 30 Guilford Street, London WC1N 1EH, United Kingdom. 